\title{
Treatment of congenital thoracic scoliosis with associated rib fusions using VEPTR expansion thoracostomy: a surgical technique
}

\author{
Romain Dayer · Dimitri Ceroni · Pierre Lascombes
}

Received: 19 April 2014/Revised: 24 April 2014/ Accepted: 24 April 2014/Published online: 14 May 2014

(C) Springer-Verlag Berlin Heidelberg 2014

\begin{abstract}
Introduction Untreated growing patients with congenital scoliosis and fused ribs will develop finally thoracic insufficiency syndrome. The technique of expansion thoracoplasty with implantation of a vertical expandable prosthetic titanium rib (VEPTR) was introduced initially to treat these children.

Methods This article attempts to provide an overview of the surgical technique of opening-wedge thoracostomy and VEPTR instrumentation in children with congenital thoracic scoliosis and fused ribs.

Results Our modification of the surgical approach using a posterior midline incision rather than the modified thoracotomy incision initially described could potentially help to diminish wound dehiscence and secondary infection, while preserving a more acceptable esthetic appearance of the back.

Conclusions Vertical expandable prosthetic titanium ribbased treatments should be undertaken only with a good knowledge of its numerous specific complications. Every aspect of the treatment should be oriented to minimize these complications. At the same time it should be kept in mind that the ultimate step of this long-term fusionless treatment strategy will be a technically demanding spine fusion.
\end{abstract}

Keywords Congenital thoracic scoliosis - Fused ribs · Thoracic insufficiency syndrome Vertical expandable prosthetic titanium rib - VEPTR $\cdot$ Expansion

R. Dayer · D. Ceroni · P. Lascombes $(\bowtie)$

Service d'orthopédie pédiatrique, Département de l'enfant et de l'adolescent, Hôpital des enfants, Hôpitaux Universitaires de Genève, Rue Willy-Donzé 6, 1211 Geneva 14, Switzerland e-mail: pierre.lascombes@hcuge.ch thoracoplasty $\cdot$ Opening-wedge thoracostomy $\cdot$ Surgical technique

\section{Introduction}

A unique method of expansion thoracoplasty (termed an opening-wedge thoracostomy) with primary longitudinal lengthening using a chest wall distractor known as vertical expandable prosthetic titanium rib (VEPTR) has been developed by Campbell and Smith since 1989 [1]. The VEPTR (Synthes Spine Co., West Chester, Pennsylvania, USA; European Patent no. 0530177) is a modular longitudinal rib-based distraction device, which is used as a ribto-rib construct, a hybrid construct (rib-to-lumbar hook) or a rib-to-pelvis construct (rib-to-Dunn McCarthy hook) [24]. It was introduced in Europe in 2002 [5]. The VEPTR opening-wedge thoracostomy in cases of thoracic congenital scoliosis associated with rib fusions is focused on the enlargement of the constricted thorax to provide room for growth of the underlying lungs, with the goal to prevent or treat the thoracic insufficiency syndrome rather than direct correction of the deformed spine alone [6]. The basic principle of this technique is first to perform an acute expansion thoracoplasty with an opening-wedge thoracostomy of fused ribs, second to stabilize the correction with the VEPTR implant during the same surgery, and finally to perform repetitive secondary lengthening and sometimes device replacement procedures to maintain deformity correction and accommodate with growth [2, 6]. Indications for expansion thoracostomy and VEPTR are conditions that progressively constrict the thoracic cage, inducing a thoracic insufficiency syndrome, defined as the inability of the thorax to support normal respiration or lung growth $[6,7]$. They can be divided into three groups [8]. The first one is 
progressive thoracic congenital scoliosis with unilateral unsegmented bars associated to fused ribs, inducing unilateral constriction of one hemithorax. Although rare, this condition will invariably progress and is the most frequently reported indication for this treatment $[1,5,6,9$ 14]. The second group of indications comprises pathologies inducing bilateral underdevelopment of the chest with a hypoplastic thorax, commonly encountered in rare conditions such as Jarcho-Levin Syndrome, Jeune's syndrome, Ellis van Creveld Syndrome, achondroplasia and VACTERL syndrome [10, 11, 14-19]. The third group includes conditions inducing a flail chest syndrome (unstable thorax due to missing ribs, congenital or acquired) $[10,14,15$, 19]. The VEPTR instrumentation without expansion thoracoplasty can also be used as an alternative to growing rods techniques for the treatment of other types of early onset scoliosis [3, 5, 20-23].

\section{Methods}

Surgical anatomy of the posterior chest wall

Knowledge of the anatomy of the muscles covering the posterior thoracic wall helps the surgeon to plane appropriately skin incisions as well as type of surgical approach. This is of primary importance, when considering that repeat exposures for rod lengthening will be needed and that implant prominence with possible wound breakdown can often be an issue during VEPTR-based treatments [24]. The posterior muscles of the chest wall are superposed in four layers [25]. The first or superficial layer consists of two large muscles covering the posterior part of the trunk. The trapezius muscle originates superiorly from the occipital bone, the spinous processes of $\mathrm{C} 2$ to $\mathrm{T} 12$, including the nuchal ligament. This large trapezoidal shape muscle inserts on the posterior spine of the scapula, the acromion, and the lateral part of the clavicle. The latissimus dorsi muscle originates inferiorly from the posterior aspect of the iliac crest and the spinous processes of S2 to T7. Its inferio-medial portion is an aponeurosis. On both sides, muscle fibers converge superiorly in a triangular fashion toward the proximal humerus to insert on the anterior aspect of the humerus on the posterior edge of the bicipital gutter. The second layer is made of two muscles from cranial to caudal. The rhomboid minor muscle runs from $\mathrm{C} 7$ to $\mathrm{T} 1$ spinous processes to the proximal fourth of the medial edge of the scapula. The rhomboid major muscle is running from $\mathrm{T} 2$ to $\mathrm{T} 5$ spinous processes to the medial edge of the scapula below the scapular spine level. The third layer consists of the two serratus posterior superior and inferior muscles, respectively, running from the spinous processes of $\mathrm{C} 6$ to $\mathrm{T} 2$ and $\mathrm{T} 11$ to $\mathrm{L} 2$ to the ribs
2-6 and 9-12. The superior is angled from midline obliquely towards lateral and distal and the inferior obliquely towards lateral and proximal. The fourth or deep layer of the posterior chest wall consists of several muscles joining in a long axial group, the erector spinae. This muscle group is further subdivided into three columns from lateral to medial running from the sacrum, the posterior iliac crest, and the vertebrae distally and inserting more proximally on the ribs and the posterior elements of the vertebrae. The iliocostalis muscles form the lateral column. The intermediate column is made by the longissimus muscles. Medially, the spinalis muscles fill the vertebral gutter (Fig. 1).

\section{Preoperative evaluation}

Patients should be accepted for surgery only after a multidisciplinary evaluation involving a pediatric orthopedic surgeon, a pediatric general surgeon and a pediatric pulmonologist [1]. Ideally the preoperative pediatric anesthesia consultation should also include the intensive care unit team. Plain chest and spine radiographs represent the first step in the understanding of the deformity. They will be better complemented by computed tomography studies to further assess the three-dimensional effect thoracic deformity including spinal rotation and loss of lung volume and for planning sites of thoracostomy and device attachment $[2,7]$. The extensive presurgical workup of patients with congenital scoliosis is dictated by the high incidence of abnormalities affecting other organ systems and the goal to diminish perioperative complications [26, 27]. Given the fact that up to $20 \%$ of these children may present with a possibly asymptomatic urologic pathology, all of them should be screened with abdominal ultrasound or by magnetic resonance imaging (MRI) of the abdomen during the time of the spine MRI [27-29]. The high frequency of associated cardiac defects warrants preoperative echocardiogram [30]. Similarly, the high incidence of neural axis abnormalities associated to congenital scoliosis makes spine MRI mandatory even in patients with normal neurological examination, especially in patients with mixed or segmentation defects [30-32]. Reduction of a spinal deformity can produce a spinal cord injury if the cord is splitted by a bony spicule (like in diastematomyelia) or tethered distally. Surgical intervention for intrathecal anomalies may therefore be needed before correction of the deformity of the spine is undertaken [27]. Given the possibility of thoracic insufficiency syndrome in the setting of congenital thoracic scoliosis associated with fused ribs, standard pulmonary function test should also be done in cooperative patients to assess restrictive lung disease, both as part of the preoperative anesthetic evaluation and as part of future assessment of treatment benefits [7]. Unfortunately such testing is seldom possible in children under 

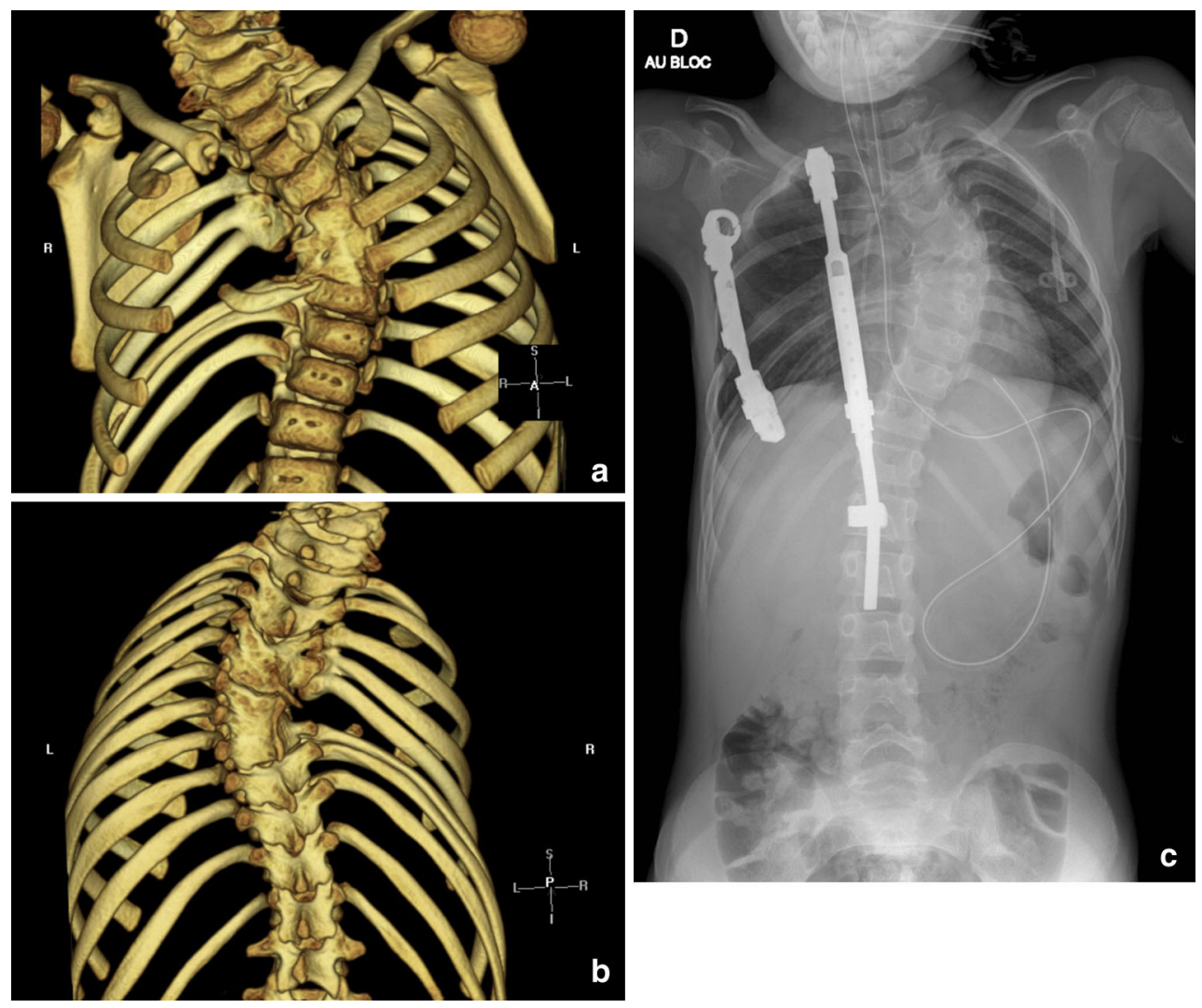

Fig. 1 a Anterior view of the three-dimensional CT scan reconstruction of a 7-year-old girl with progressive thoracic congenital scoliosis and fused ribs on the concave side of the curve. Defects of segmentation with multiple misshapen vertebrae fused on the convex side and defects of formation with missing vertebrae on the concave side are present at the apex. On the right side, medial fusion is present between ribs 4 and 5 somewhat cephalad to the apex, ribs 6 and 7 are missing concomitantly with the vertebral formation defect, and ribs 8 and 9 are completely fused. b Posterior view of the three-dimensional

5 years of age [33]. Finally, preoperative correction of possible nutritional deficiencies is suggested as of primary importance in an attempt to decrease the risk of wound problems and infection associated both to the initial VEPTR procedure as well as multiple subsequent lengthening surgeries [20]. This may require supplemental nutrition or percutaneous gastrostomy in some patients [10].

Surgical technique of VEPTR expansion thoracostomy for congenital thoracic scoliosis and fused ribs

\section{Patient positioning}

The surgery was initially described with the patient under general anesthesia installed in a lateral decubitus position with the side of the concave hemithorax upward $[2,8,10]$. We prefer the prone position because we use a midline spinal incision for the whole exposure in contrast to
CT scan reconstruction of the same patient with mixed vertebral defects. c She was treated with opening-wedge thoracostomies at two levels and instrumentation with a lateral thoracic and a medial hybrid vertical, expandable titanium rib. The lumbar extension of the hybrid implant was mated to a laminar hook at L1. Intraoperative radiograph demonstrating immediate increase in space available for the lung of the concave hemithorax and partial indirect correction of the scoliotic curve

Campbell et al. [2]. An arterial line and a central venous catheter are both inserted. Prophylactic antibiotics are given intravenously $30 \mathrm{~min}$ prior to incision. Intraoperative monitoring includes upper and lower extremities somatosensory and transcranial motor-evoked potentials as well as upper extremity pulse oximetry on the side of the operation $[2,10]$. Intraoperative neuromonitoring should also be used during secondary lengthening and revision procedures [24]. The upper limbs are draped out of the surgical field as during a posterior spine fusion (Fig. 2).

\section{Exposure and soft tissues handling}

The surgical exposure should allow access to the entire posterior thoracic wall for VEPTR instrumentation and expansion opening-wedge thoracostomy. The incision must also be planned with the need of repeat exposures for rod lengthening in mind [8]. Campbell described the approach for the rib-to-rib implant insertion and thoracostomy(ies) 

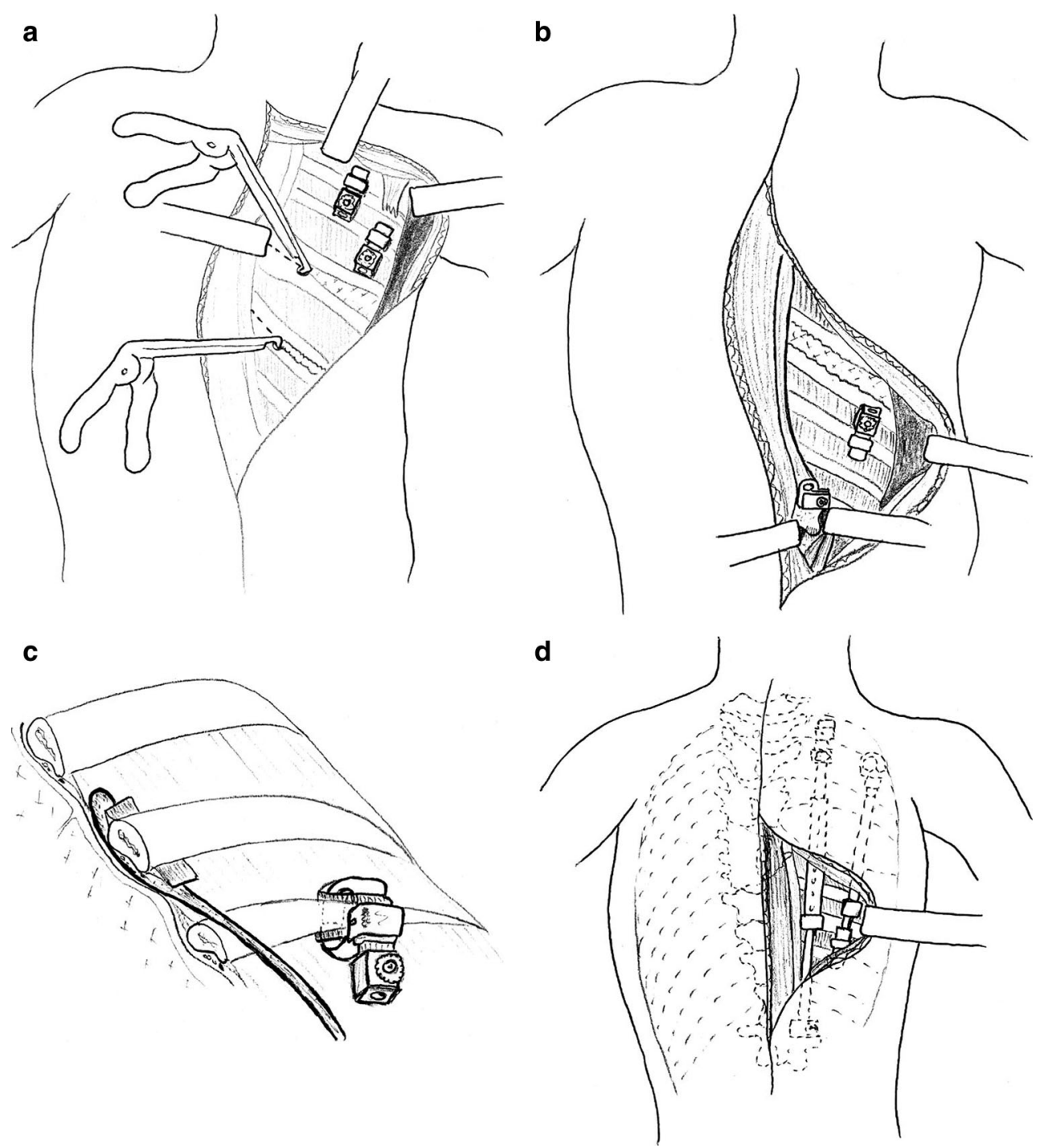

Fig. 2 a The patient is placed in the prone position and a posterior spine midline incision is made. After opening of the superficial fascia, the three superficial muscle layers of the posterior thoracic wall are detached from the spinous processes on the side of hemithorax constriction. The scapula and muscle layers are retracted alternatively superiorly and inferiorly. Medial and lateral proximal rib cradles are, respectively, inserted around the second and the third ribs in this example. The posterior scalene muscle represents the upper lateral limit of the exposure because of the neurovascular bundle. The thoracostomies are then performed. b The lateral distal rib cradle is inserted, and fixation of the lumbar extension of the hybrid rib

prosthesis is made with a laminar hook on L1 in this example. c Preparation of the site for rib cradle insertion with transverse incision in the intercostal muscles above and below the selected rib without disrupting the pleura and the neurovascular bundle. An elevator is then passed from caudad to cephalad. d Final view after thoracostomies and VEPTR instrumentation. The entire procedure has been performed through the midline skin incision. The same incision will be partially used for secondary lengthening and exchange procedures and the muscles will be detached in the same manner as during the initial surgery

with a modified thoracotomy skin incision which is made $4 \mathrm{~cm}$ caudad to the tip of the scapula and carried anteriorly in an "L" shape [2]. The trapezius and rhomboid superiorly and the latissimus dorsi muscle inferiorly are then divided in line with the skin incision. The interval between the scapula and the chest wall is developed superiorly. The paraspinous muscles are finally reflected from lateral to medial to the tips of the transverse processes with care taken not to damage rib periosteum or to expose the spine,

which could provoke fusion [2]. Other authors modified this approach by extending the incision slightly more caudad in a "J" shape, with deep incision in the latissimus dorsi more distal than the skin incision, and sometimes "delay" of the flap in the case of multiple prior thoracotomy scars [10]. In both cases, a separate midline lumbar incision is needed for the distal anchorage of the rib-tospine device $[2,10]$. Incisions and muscle flaps must be planned to allow adequate exposure to implant insertion 
sites and thoracostomy but also to minimize the surgical wound overlying the most prominent portions of the VEPTR [20]. A modified thoracotomy "L"- or "J"-shaped incision will inevitably cross the implant at some point. For these reasons, we favor a posterior spine incision for implant insertion and thoracostomy. First the skin incision is made along the midline over the spinous processes from slightly cephalad to the level chosen for superior implant insertion to slightly caudad to the level chosen for the distal rib cradle (or lumbar extension). The subcutaneous layer is incised in line with the skin incision, as well as the thoracodorsal fascia. Then care is taken to not open the spinous process apophyses. The three most superficial layers of muscles are detached with electrocautery close to their insertion on the posterior vertebral elements in a strict extraperiosteal manner to not promote spine fusion. Deeply the erector spinae is left intact on the posterior vertebral elements. The dissection is carried extraperiosteally over the tip of the transverse processes and then over the ribs.

\section{Superior implant insertion}

The site for proximal rib cradle insertion is then prepared. The selected level should be well within the cephalad part of the curve but not proximal to the curve to not increase a compensatory proximal curve without correcting the primary curve. Usually, this corresponds to the rib at the level of the end vertebra of the scoliotic curve. The chosen rib also has to be at least $1-\mathrm{cm}$ thick otherwise two ribs have to be attached by the rib cradle. The first rib is avoided because of the risk of brachial plexus injury. The insertion site will have the best mechanical effect if its position is just adjacent to the tips of the transverse processes [2]. Two transverse $1.5 \mathrm{~cm}$ incisions are made with the electrocautery in the intercostal muscles above and below the selected rib, and an elevator is passed from caudad to cephalad without disrupting the pleura and the neurovascular bundle. The cephalad rib cradle is inserted and locked.

\section{Inferior implant insertion}

For a rib-to-rib construct, the instrumentation at the site for the distal rib cradle proceeds in the same way and is inserted around one or more stable ribs. Usually the inferior rib cradle is inserted after the primary expansion thoracoplasty. In children older than 18 months, a medial hybrid construct (rib-to-spine) is usually used in conjunction with the thoracic (rib-to-rib) implant, acting as a "share-load" construct [2]. Its distal attachment on a neutral lumbar vertebra is made with a laminar hook or ideally a pedicle screw, for increased stability of distal fixation. Preoperative planning with computed tomography helps the surgeon to assess either the size of the canal for hook placement or the size of the pedicle for screw insertion. The thoracic prosthesis (rib-to-rib) is placed secondarily lateral to the hybrid one in the posterior axillary line to help support the correction. When the rib-to-rib implant is inserted in this more lateral position, it should not be inserted more proximal than the third rib, to avoid lesion of the brachial plexus [2].

\section{Opening-wedge thoracostomy}

After insertion of the superior rib cradle, the primary expansion thoracostomy is performed. It is planned in the apex of thoracic constriction of fused ribs. Number and location of thoracostomies depend of the location of hemithoracic constriction, location of rib fusions, and secondarily of the location of spinal deformity or congenital anomalies [10]. The fibrous band lying anteriorly or posteriorly to the fusion mass is first released with electrocautery without opening the pleura. Then the fusion mass itself is osteotomised gradually with a Kerrison rongeur after opening of the periosteum, and a bone spreader is inserted and widened progressively. During this widening, the underlying pleura is bluntly released from under the ribs proximally and distally. Sometimes completion of the thoracostomy implies resection of a fusion mass that is extending medially to the transverse process and has to be released under direct vision in a medial to lateral direction after resection of the costotransverse ligament. The thoracostomy interval is further gradually widened with the bone spreader. Once the proximal ribs are pushed in a horizontal position, the correction is maintained with a rib retractor so that the distance between the superior and inferior insertion site of the VEPTR is measured. The matching rib sleeve is selected and placed over the inferior rib cradle and connected to the superior cradle to maintain the obtained opening-wedge correction. If the thoracostomy interval is larger than $3 \mathrm{~cm}$ and likely to cause a flail chest, a section of fused ribs from the proximal or distal border of the thoracostomy interval can be cut transversally and translated into the defect, a procedure termed centralization transport by Campbell et al. [2]. The rib section is sutured to the VEPTR using non-absorbable suture.

\section{Closure and postoperative care}

Pleural tears inferior to $2 \mathrm{~cm}$ are not repaired [2]. In cases of pleural defect greater than $2 \times 2 \mathrm{~cm}$, risk of herniation of the lung with projection of a lobe through the defect, significant diminution of the chest wall musculature or risk of injury to the lung at reoperation, a non-synthetic biodegradable patch material is implanted for coverage of the defect and sutured to the surrounding pleura and/or muscle, as well as the overlying VEPTR, using an absorbable suture (2 Zero, Vicryl). Intrapleural and extrapleural drains 
should be placed in these cases [19]. For wound closure, Campbell emphasizes first on the need to stretch all muscle and skin edges manually to obtain coverage [2]. All incised muscle layers are then sutured back to the median line incision with monofilament absorbable sutures. Using this midline spine approach, the scapula is brought back in its position indirectly with muscle layers closure, and there is no need for direct fixation at the inferior tip of the scapula, as proposed by Campbell. However, acute iatrogenic thoracic outlet syndrome is still possible with this technique at time of closure, and consequently the change in pulse oximeter readings or in ulnar nerve tracings on the side of the operation should be carefully observed. Subcutaneous layer and skin are closed using monofilament absorbable suture. The choice of monofilament suture is dictated by the need to minimize inflammation and foreign body reaction at the site of wound closure. Polyurethane foam protectors are used to protect the skin over prominent implants in the early postoperative phase. All patients are admitted to the pediatric intensive care unit and remain initially intubated. No brace is required postoperatively. Return to full activities is allowed 6 weeks after surgery.

\section{Device lengthening and replacement procedures}

The devices are expanded electively at intervals of 6 months following initial implantation. Prophylactic antibiotics are given intravenously. In every case, the intraoperative neuromonitoring should be used. Positioning is the same as that of the first surgery. The distraction lock is exposed using part of the prior midline incision and is removed. The device is then lengthened slowly during 5-10 $\mathrm{mm}$ until the reactive force becomes considerable, to avoid rib fracture and acute fixation loosening [2]. The medial rib prosthesis should be lengthened first followed by the lateral one.

Replacement of the implants is performed using part of the same posterior midline approach for change-out of the superior and inferior cradle, lumbar extension, and the rib sleeve. We feel that using this posterior midline approach for initial implantation and iterative lengthening or secondary exchange procedures offers the advantage of not ending with the incision overlying the implants, thereby potentially diminishing the risk of wound dehiscence and infection. It also probably gives a superior cosmetic effect to the back rather multiple limited incisions and ultimately the final fusion will be performed through the same posterior spine approach [34].

\section{Results}

This fusionless treatment was associated with mid-term follow-up in growing patients with congenital scoliosis and fused ribs to preserve the longitudinal growth of both the concave and convex sides of the thoracic spine and unilateral unsegmented bars, as well as with significant improvement of Cobb angle, lateral deviation of the spine, space available for the lung, cervical tilt and shoulder balance [1, 6, 9, 13]. Even if it sounds likely that a longer thoracic cage should provide increased volume for the growth of the underlying lungs with potential clinical benefit [6], to date there is conflicting evidence that this treatment is associated with significant improvement in lung function and volume [11, 14, 35, 36].

\section{Complications}

Vertical expandable prosthetic titanium rib-based treatments unfortunately have many potential complications during their whole course, from the initial implantation to the final fusion. They are inherent to iterative surgeries on growing children with spine deformity [20]. A comprehensive knowledge of these associated problems is essential for the surgeon, both for adequate preoperative information of the patient's family and for adopting treatment strategies aimed at minimizing complications. Reported complications include frequent wound dehiscence and secondary deep infections, anchor point problems with acute rib fracture or chronic implant migration, neurologic issues, mainly brachial plexus palsy or acute thoracic outlet syndrome, chest wall stiffness and reoccurrence of rib fusions, spine autofusions, kyphosis and sagittal plane imbalances, and occasionally shoulder stiffness as well as spontaneous fusion of the scapula to the implant [1, 4-6, 9, 10, 37]. Ultimately, it should be kept in mind that the final step (i.e. definitive spine fusion) of this difficult long-term treatment is further rendered challenging and risky for the patient by these secondary problems, with a potentially less than satisfactory end result [34].

\section{Discussion}

Vertical expandable prosthetic titanium rib-based treatments associated to thoracostomy, although they carry a significant rate of complications even in experienced hands, appear as an appropriate surgical treatment option for early onset scoliosis associated to rib fusion, with the primary goal being focused on the enlargement of the constricted thorax rather than direct correction of the scoliosis alone [23]. Treatments using VEPTR implants without expansion thoracoplasty have been reported as an alternative to growing rods techniques for the treatment of other types of early onset scoliosis [3, 5, 20-23]. Among the different indications reported, we feel that the more valid is probably for the surgical treatment of a progressive 
spinal deformity presenting in a skeletally immature patient with spina bifida [3]. Bilateral VEPTR rib-to-pelvis instrumentation in this case represent an interesting treatment option because it offers the possibility to obtain solid anchorage even in the absence of posterior spinal element. It also address the correction of pelvic obliquity often encountered in this patients population. Finally, the surgical approach and implants stay away from the myelomeningocele scar at the midline thoraco-lumbar and lumbar area. Rib anchorage on both sides can be made through a single posterior midline extraperiosteal approach in the upper thoracic area. Distally the pelvic hooks are inserted though two posterior and lateral approaches with incisions made close to the level of the ilium. Finally, the assembled proximal and distal extensions are inserted in a submuscular fashion from distant incision sites, thereby keeping the implant far from midline at the level of the myelomeningocele closure site, minimizing the risk of skin breakdown and secondary infection. However, we think that for the surgical treatment of other types of early onset scoliosis and especially in the idiopathic patients, the VEPTR instrumentation does not represent the ideal implant. Its usage as an alternative to classic growing rod constructs makes little sense, since there is no need to anchor on the thoracic cage if this anatomic area is not primarily affected by the pathology [23]. In addition, bilateral rib-to-pelvis technique with the VEPTR has been associated with increased incidence of crouched gait, so that this treatment option should not be considered in ambulatory children [37]. In case of pelvic obliquity, dual iliac fixation with screws or rods has been shown to provide effective deformity correction and to achieve growth [38]. The last disadvantage for early onset scoliosis treatment of the VEPTR implants compared to growing rods is linked to the size of the implants; most pediatric deformity implant sets currently available have a significantly lower profile design, minimizing the risk of complications linked to implants prominence. Because of all these elements taken together, we tend to restrict the use of VEPTR implants in our practice to these two groups of indication, namely congenital thoracic scoliosis associated with rib fusions and spinal deformity encountered in immature non-ambulating patients with myelodysplasia.

\section{Conclusions}

Vertical expandable prosthetic titanium rib-based treatments should be undertaken only by trained surgeons with a good knowledge of its numerous specific complications. Every aspect of the treatment should be oriented with the goal to minimize these complications. Regarding the surgical approach, we feel that using a single midline spine incision, instead of the initially described modified thoracotomy approach, may potentially lower the risk of wound dehiscence and hence of secondary deep infection. Along the same line, the development of low profile implants could also lesser implant prominence in small children. Second generation VEPTR is available. The interesting modifications include first the possibility to bend the cranial part of the proximal extension to better fit to each patient anatomy and second the option of proximal insertion on two adjacent or non-adjacent ribs. However, implant profile could probably still be reduced. Another implant development strategy would be to introduce some flexibility in the proximal and distal extension. A rib-tospine construct act as a solid bridge between a mobile rib and a fixed spinal anchor. A decrease of implant stiffness could make it accommodate to some of the relative motion of the upper thorax, and hence possibly decrease the rate of proximal and distal anchor point problem.

Acknowledgments The authors would like to thank Prof. Carol C. Hasler (Orthopaedic Department, University Children's Hospital, Basel, Switzerland) for his experienced teaching of the VEPTR surgical technique.

Conflict of interest Authors do not have any conflicts of interest relevant to this article to report.

Financial support None of the authors received financial support for this study.

\section{References}

1. Campbell RM Jr, Smith MD, Mayes TC et al (2004) The effect of opening wedge thoracostomy on thoracic insufficiency syndrome associated with fused ribs and congenital scoliosis. J Bone Joint Surg Am 86-A:1659-1674

2. Campbell RM Jr, Smith MD, Hell-Vocke AK (2004) Expansion thoracoplasty: the surgical technique of opening-wedge thoracostomy. Surgical technique. J Bone Joint Surg Am 86-A(Suppl 1):51-64

3. Flynn JM, Ramirez N, Emans JB et al (2011) Is the vertebral expandable prosthetic titanium rib a surgical alternative in patients with spina bifida? Clin Orthop Relat Res 469:1291-1296

4. Hasler CC, Mehrkens A, Hefti F (2010) Efficacy and safety of VEPTR instrumentation for progressive spine deformities in young children without rib fusions. Eur Spine J 19:400-408

5. Hell AK, Campbell RM, Hefti F (2005) The vertical expandable prosthetic titanium rib implant for the treatment of thoracic insufficiency syndrome associated with congenital and neuromuscular scoliosis in young children. J Pediatr Orthop B 14:287-293

6. Campbell RM Jr, Hell-Vocke AK (2003) Growth of the thoracic spine in congenital scoliosis after expansion thoracoplasty. J Bone Joint Surg Am 85-A:409-420

7. Campbell RM Jr, Smith MD, Mayes TC et al (2003) The characteristics of thoracic insufficiency syndrome associated with fused ribs and congenital scoliosis. J Bone Joint Surg Am 85-A:399-408

8. Lewandrowski KU, Cambell RM, Emans JB (2001) Vertical rib expansion for thoracic insufficiency syndrome indications and technique. Orthop J Harv Med Sch 3:65-73 
9. Campbell RM Jr, Adcox BM, Smith MD et al (2007) The effect of mid-thoracic VEPTR opening wedge thoracostomy on cervical tilt associated with congenital thoracic scoliosis in patients with thoracic insufficiency syndrome. Spine 32:2171-2177

10. Emans JB, Caubet JF, Ordonez CL et al (2005) The treatment of spine and chest wall deformities with fused ribs by expansion thoracostomy and insertion of vertical expandable prosthetic titanium rib: growth of thoracic spine and improvement of lung volumes. Spine 30:S58-S68

11. Gadepalli SK, Hirschl RB, Tsai WC et al (2011) Vertical expandable prosthetic titanium rib device insertion: does it improve pulmonary function? J Pediatr Surg 46:77-80

12. Hell AK, Hefti F, Campbell RM (2004) Behandlung der kongenitalen skoliose mit dem vertical-expandable-prosthetic-titaniumrib-Implant. Orthopaedics 33:911-918

13. Samy MA, Al Zayed ZS, Shaheen MF (2009) The effect of a vertical expandable prosthetic titanium rib on shoulder balance in patients with congenital scoliosis. J Child Orthop 3:391-396

14. Waldhausen JH, Redding GJ, Song KM (2007) Vertical expandable prosthetic titanium rib for thoracic insufficiency syndrome: a new method to treat an old problem. J Pediatr Surg 42:76-80

15. Gill R, Kinsella CR Jr, Lin AY et al (2011) Acellular dermal matrix in the treatment and prevention of exposed vertical expandable prosthetic titanium ribs. Spine 36:E563-E567

16. Lacher M, Dietz HG (2011) VEPTR (Vertical Expandible Prosthetic Titanium Rib) treatment for Jeune syndrome. Eur J Pediatr Surg 21:138-139

17. Ramirez N, Flynn JM, Emans JB et al (2010) Vertical expandable prosthetic titanium rib as treatment of thoracic insufficiency syndrome in spondylocostal dysplasia. J Pediatr Orthop 30: $521-526$

18. Reinker K, Simmons JW, Patil V et al (2011) Can VEPTR ${ }^{(\mathrm{R})}$ control progression of early-onset kyphoscoliosis? A cohort study of $\operatorname{VEPTR}^{(\mathrm{R})}$ patients with severe kyphoscoliosis. Clin Orthop Relat Res 469:1342-1348

19. Smith MD, Campbell RM (2006) Use of a biodegradable patch for reconstruction of large thoracic cage defects in growing children. J Pediatr Surg 41:46-49

20. Akbarnia BA, Emans JB (2010) Complications of growth-sparing surgery in early onset scoliosis. Spine 35:2193-2204

21. Greggi T, Lolli F, Di Silvestre M et al (2012) Complications incidence in the treatment of early onset scoliosis with growing spinal implants. Stud Health Technol Inform 176:334-337

22. Latalski M, Fatyga M, Gregosiewicz A (2011) Problems and complications in VEPTR-based treatment. Ortop Traumatol Rehabil 13:449-455
23. Yazici M, Emans J (2009) Fusionless instrumentation systems for congenital scoliosis: expandable spinal rods and vertical expandable prosthetic titanium rib in the management of congenital spine deformities in the growing child. Spine 34:1800-1807

24. Sankar WN, Acevedo DC, Skaggs DL (2010) Comparison of complications among growing spinal implants. Spine 35: 2091-2096

25. Testut L (1896) Traité d'anatomie humaine, 3rd edn. Octave Doin, Paris

26. Beals RK, Robbins JR, Rolfe B (1993) Anomalies associated with vertebral malformations. Spine 18:1329-1332

27. Hedequist D, Emans J (2004) Congenital scoliosis. J Am Acad Orthop Surg 12:266-275

28. Drvaric DM, Ruderman RJ, Conrad RW et al (1987) Congenital scoliosis and urinary tract abnormalities: are intravenous pyelograms necessary? J Pediatr Orthop 7:441-443

29. MacEwen GD, Winter RB, Hardy JH (1972) Evaluation of kidney anomalies in congenital scoliosis. J Bone Joint Surg Am 54:1451-1454

30. Basu PS, Elsebaie H, Noordeen MH (2002) Congenital spinal deformity: a comprehensive assessment at presentation. Spine 27:2255-2259

31. McMaster MJ (1984) Occult intraspinal anomalies and congenital scoliosis. J Bone Joint Surg Am 66:588-601

32. Suh SW, Sarwark JF, Vora A et al (2001) Evaluating congenital spine deformities for intraspinal anomalies with magnetic resonance imaging. J Pediatr Orthop 21:525-531

33. England SJ (1988) Current techniques for assessing pulmonary function in the newborn and infant: advantages and limitations. Pediatr Pulmonol 4:48-53

34. Lattig F, Taurman R, Hell AK (2012) Treatment of Early Onset Spinal Deformity (EOSD) with VEPTR: a challenge for the final correction spondylodesis: a case series. J Spinal Disord Tech [Epub ahead of print]

35. Mayer OH, Redding G (2009) Early changes in pulmonary function after vertical expandable prosthetic titanium rib insertion in children with thoracic insufficiency syndrome. J Pediatr Orthop 29:35-38

36. Motoyama EK, Yang CI, Deeney VF (2009) Thoracic malformation with early-onset scoliosis: effect of serial VEPTR expansion thoracoplasty on lung growth and function in children. Paediatr Respir Rev 10:12-17

37. Smith JT (2011) Bilateral rib-to-pelvis technique for managing early-onset scoliosis. Clin Orthop Relat Res 469:1349-1355

38. Sponseller PD, Yang JS, Thompson GH et al (2009) Pelvic fixation of growing rods: comparison of constructs. Spine 4(16): $1706-1710$ 